\title{
What is the Higgs phase of a gauge Higgs theory?
}

\section{Jeff Greensite ${ }^{* \dagger}$}

San Francisco State University

E-mail: greensitesfsu.edu

\section{Kazue Matsuyama}

San Francisco State University

E-mail: kazuemesfsu.edu

\begin{abstract}
We argue that there is an essential and gauge invariant distinction between the Higgs and confinement phases of a gauge Higgs theory, in terms of both symmetry and type of confinement, when the scalar field is in the fundamental representation of the gauge group. The Higgs phase is the spin glass phase of a gauge Higgs theory, in which custodial symmetry and a global center subgroup of the gauge symmetry are spontaneously broken. Although the asymptotic spectrum consists of color singlets in both the confinement and Higgs phases, the confinement phase satisfies a generalization of the Wilson area law criterion which we call "separation of charge" confinement, while the Higgs (spin glass) phase does not.
\end{abstract}

37th International Symposium on Lattice Field Theory- LATTICE 2019

June 16-22 2019, Wuhan, China

\footnotetext{
* Speaker.

${ }^{\dagger}$ Work supported by the US Department of Energy under Grant No. DE-SC0013682.
} 
There is no thermodynamic phase transition which entirely separates the Higgs and confinement phases of an SU(2) gauge Higgs theory in 3+1 dimensions [1,2], and the particles in the asymptotic spectrum are color singlets throughout the phase diagram, created by gauge invariant local composite operators $[3,4]$. In addition, the existence of a non-vanishing Higgs expectation value is gauge dependent [5]. These facts, combined with the Elitzur theorem, tend to discourage any attempt to distinguish between the Higgs and confinement-like regions of a gauge Higgs theory; these regions are generally viewed as part of the same phase. In these proceedings we will contest that view. We will argue that the transition between the confinement and Higgs phases in a gauge Higgs system is as meaningful as the spin glass transition in a spin system, in fact it is a type of spin glass transition, and as such it is characterized by the breaking of a global symmetry detected by a gauge invariant order parameter. While the spectrum in both the confinement and Higgs phases consists of particles created by local gauge invariant operators, there is nonetheless a physical distinction between these phases, generalizing the distinction between an area vs. perimeter law falloff for Wilson loops in a pure gauge theory. A more detailed presentation of these results can be found in ref. [6].

The Edwards-Anderson model of a spin glass [7] is based on the following Hamiltonian

$$
H_{\text {spin }}=-\sum_{i j} J_{i j} s_{i} s_{j}-h \sum_{i} s_{i}
$$

where the $J_{i j}$ are random couplings, drawn from some probability distribution $P(J)$, and the $s_{i}= \pm 1$ are Ising spins. At $h \rightarrow 0$ the model is obviously invariant under the global symmetry $s_{i} \rightarrow z s_{i}, z \in Z_{2}$, but in general the spatial average of $s_{i}$ vanishes, due to the random couplings. It might be that the expectation value of a spin $s_{i}$ at a particular site $i$ is non-zero, but this would also vanish upon averaging over the random couplings. Despite this fact there is a way to characterize the spontaneous breaking of the global $Z_{2}$ symmetry. Define

$$
\begin{aligned}
Z_{\mathrm{spin}}(J) & =\sum_{\{s\}} e^{-H_{\mathrm{spin}} / k T}, \bar{s}_{i}(J)=\frac{1}{Z_{\mathrm{spin}}(J)} \sum_{\{s\}} s_{i} e^{-H_{\mathrm{spin}} / k T} \\
q(J) & =\frac{1}{V} \sum_{i} \bar{s}_{i}^{2}(J),\langle q\rangle=\int \prod_{i j} d J_{i j} q(J) P(J),
\end{aligned}
$$

where $q(J)$ is the Edwards-Anderson order parameter [7]. When the expectation value $\langle q\rangle$ is nonzero in the infinite volume $V \rightarrow \infty$ and $h \rightarrow 0$ limits, the system is in the spin glass phase, and the $Z_{2}$ global symmetry is spontaneously broken.

We will illustrate the corresponding construction in SU(2) gauge Higgs theory with a unimodular Higgs field $\phi$, although most of what we discuss is readily generalized to $\mathrm{U}(1)$ and $\mathrm{SU}(N>2)$ theories. The action is

$$
S=-\beta \sum_{\text {plaq }} \frac{1}{2} \operatorname{Tr}\left[U_{\mu}(x) U_{v}(x+\hat{\mu}) U_{\mu}^{\dagger}(x+\hat{v}) U_{v}^{\dagger}(x)\right]-\gamma \sum_{x, \mu} \frac{1}{2} \operatorname{Tr}\left[\phi^{\dagger}(x) U_{\mu}(x) \phi(x+\widehat{\mu})\right],
$$

where $\phi$ is an $\mathrm{SU}(2)$ group-valued field. This theory has the following invariances:

$$
U_{\mu}(x) \rightarrow L(x) U_{\mu}(x) L^{\dagger}(x+\hat{\mu}), \phi(x) \rightarrow L(x) \phi(x) R,
$$


where $L(x) \in \mathrm{SU}(2)_{\text {gauge }}$ is a local gauge transformation, while $R \in \mathrm{SU}(2)_{\text {global }}$ is a global transformation belonging to the custodial symmetry group. A custodial symmetry is a symmetry of the action under transformations of the scalar field alone, the group in this case is $\mathrm{SU}(2)$. Let

$$
\begin{aligned}
\exp [-H(\phi, U) / k T] & =\left\langle\phi, U\left|e^{-H / k T}\right| \phi, U\right\rangle \\
& =\sum_{n}\left|\Psi_{n}(\phi, U)\right|^{2} e^{-E_{n} / k T},
\end{aligned}
$$

where the $\Psi_{n}$ are energy eigenstates. $H(\phi, U)$ has a vast number of near-degenerate minima at any fixed $U$. In analogy to spin models, we insert a small custodial symmetry breaking term

$$
H_{\text {spin }}(\phi, U, \eta)=H(\phi, U)-h \sum_{\mathbf{x}} \operatorname{Tr}\left[\eta^{\dagger}(\mathbf{x}) \phi(\mathbf{x})\right]
$$

with $\eta(\mathbf{x})$ an SU(2)-valued field. We then define

$$
\begin{aligned}
Z_{\text {spin }}(U, \eta) & =\int D \phi(\mathbf{x}) e^{-H_{\text {spin }}(\phi, U, \eta) / k T}, \bar{\phi}(\mathbf{x} ; U, \eta)=\frac{1}{Z_{\text {spin }}(U, \eta)} \int D \phi \phi(\mathbf{x}) e^{-H_{\text {spin }}(\phi, U, \eta) / k T} \\
\Phi(U) & =\frac{1}{V}\left[\sum_{\mathbf{x}} \mid \bar{\phi}(\mathbf{x} ; U, \eta \mid]_{\eta \in \mathcal{N}(U)},\langle\Phi\rangle=\int D U_{i}(\mathbf{x}) \Phi(U) P(U),\right.
\end{aligned}
$$

which should be compared to eqs. (2). $P(U)$ is a gauge invariant probability distribution for the link variables, described below, which is obtained from the partition function after integrating out the scalar field. The expression $\mathcal{N}(U)$ represents a set $\eta(\mathbf{x})$ fields defined by

$$
\mathcal{N}(U)=\underset{\eta}{\arg \max } \sum_{\mathbf{x}}\left|\int D \phi \phi(\mathbf{x}) e^{-H_{\text {spin }}(\phi, U, \eta) / k T}\right|,
$$

and the elements of this set at a given $U$ transform into one another by custodial transformations. It can be shown that $Z_{\text {spin }}(U) \equiv Z_{\text {spin }}(U, \eta \in \mathcal{N}(U))$ and the order parameter $\Phi(U)$ are both gauge invariant, even at finite $h$, and independent of the choice of $\eta \in \mathcal{N}(U)$.

$P(J)$ in a spin glass, for a pair of sites $i, j$, is typically drawn from a product of Gaussian distributions for each $J_{i j}$, or else $J_{i j}= \pm J$ with equal probability. In a gauge Higgs theory we find $P(U)$ from the condition that expectation value of some gauge invariant operator $Q(U)$ is given by

$$
\begin{aligned}
\langle Q\rangle & =\frac{\operatorname{Tr} Q e^{-H_{\text {spin }} / k T}}{\operatorname{Tr} e^{-H_{\text {spin }} / k T}} \\
& =\frac{1}{Z} \int D U_{i}(\mathbf{x}) Q(U) \int D \phi(\mathbf{x}) e^{-H_{\text {spin }}(\phi, U, \eta \in \mathcal{N}(U)) / k T} \\
& =\frac{1}{Z} \int D U_{i}(\mathbf{x}) Q(U) Z_{\text {spin }}(U) \\
& =\int D U_{i}(\mathbf{x}) Q(U) P(U),
\end{aligned}
$$

and therefore

$$
P(U)=\frac{Z_{\text {spin }}(U)}{Z} .
$$

With this choice, $\langle Q\rangle$ agrees with the standard expectation value of $Q$, at $h \rightarrow 0$, in a gauge Higgs theory. The criterion for spontaneously broken custodial symmetry is then

$$
\lim _{h \rightarrow 0 V \rightarrow 0} \lim _{V \rightarrow}\langle\Phi\rangle\left\{\begin{array}{l}
=0 \text { unbroken symmetry } \\
>0 \text { broken symmetry }
\end{array},\right.
$$




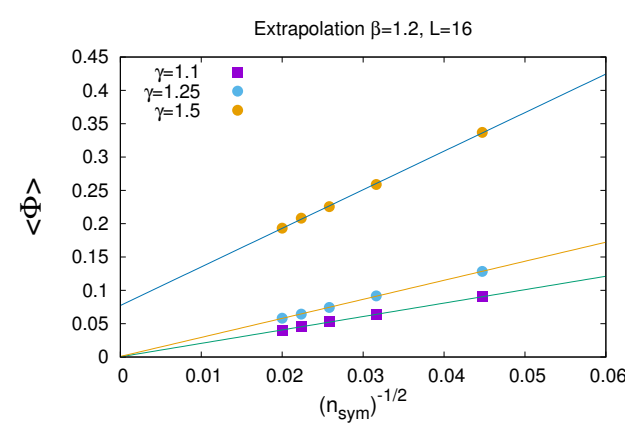

(a)

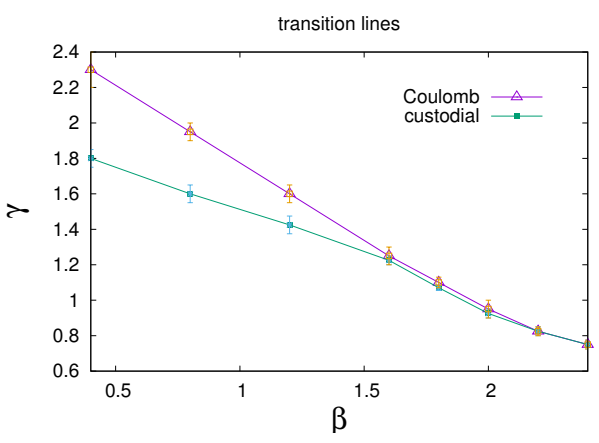

(b)

Figure 1: (a) Extrapolation of $\langle\Phi\rangle$ to $n_{\text {sym }} \rightarrow \infty$ above $(\gamma=1.5)$ and below $(\gamma=1.1,1.25)$ the custodial symmetry breaking transition at $\beta=1.2, \gamma=1.4$, in SU(2) gauge Higgs theory. The lattice volume is $16^{4}$; error bars are smaller than the symbol sizes. (b) Transition lines above which (i) $\left|\langle\phi\rangle_{C}\right|>0$ in Coulomb gauge (triangular data points), and (ii) $\langle\Phi\rangle>0$, i.e. broken custodial symmetry (filled circles). Note that the region where $|\langle\phi\rangle|>0$ lies entirely within the broken custodial symmetry phase, as it must from the bound derived below.

which is entirely analogous to the Edwards-Anderson spin glass criterion

$$
\lim _{h \rightarrow 0 V \rightarrow 0}\langle q\rangle\left\{\begin{array}{l}
=0 \text { non-spin glass phase } \\
>0 \text { spin glass phase }
\end{array} .\right.
$$

The spin glass order parameter $\Phi$ is evaluated numerically as follows: A Monte Carlo simulation of the SU(2) gauge Higgs theory (3) is carried out in the usual way. The data taking sweep is actually a series of $n_{\text {sym }}$ sweeps, holding the spacelike links $U_{i}(\mathbf{x}, t=0)$ fixed on the $t=0$ time slice, but updating all other variables. Denoting by $\phi(\mathbf{x}, 0, n)$ the Higgs field at time $t=0$ on the $\mathrm{n}$-th sweep, and dispensing with $\eta$, we compute

$$
\bar{\phi}(\mathbf{x}, U)=\frac{1}{n_{s y m}} \sum_{n=1}^{n_{s y m}} \phi(\mathbf{x}, 0, n),
$$

with $\Phi(U)$ from (7). Averaging $\Phi(U)$ over all data-taking sweeps of this kind gives an estimate for $\left\langle\Phi\left(n_{\text {sym }}\right)\right\rangle$. Carrying out such simulations at a variety of $n_{\text {sym }}$ values, and fitting the data to

$$
\left\langle\Phi\left(n_{\text {sym }}\right)\right\rangle=\langle\Phi\rangle+\frac{\kappa}{\sqrt{n_{\text {sym }}}},
$$

gives an estimate for $\langle\Phi\rangle$. To determine the transition line we fix $\beta$ and vary $\gamma$, until the extrapolated value for $\langle\Phi\rangle$ begins to move away from zero. An example of the data at $\beta=1.2$ and three values of $\gamma$ is shown in Fig. 1(a). The custodial symmetry breaking transition line, joining transition points determined as just described, is shown in Fig. 1(b), which also displays the Coulomb gauge transition line above which $|\langle\phi\rangle|$ is non-zero.

Spontaneous breaking of custodial symmetry has the following properties: Consider $\langle\phi\rangle_{F}$ evaluated in a class of physical gauges defined by conditions of the form $F(U)=0$. The conditions are imposed on spacelike link variables on each time slice, removing all local gauge symmetry, but leaving some remnant global symmetry on a given time slice, as in Coulomb gauge or a maximally 
fixed axial gauge. We will refer to these as " $F$-gauges," and they have the property that the field operators $q, \bar{q}, \phi$, operating on the ground state, create physical states. We can prove that [6]

1. for all physical $F$-gauges, $\langle\Phi\rangle \geq|\langle\phi\rangle|_{F}$; and

2. there exists at least one $F$ gauge such that $\langle\Phi\rangle=\langle\phi\rangle_{F}$.

This means that broken custodial symmetry is a necessary condition for $\langle\phi\rangle_{F} \neq 0$ in any $F$-gauge, and a sufficient condition for $\langle\phi\rangle_{F} \neq 0$ in at least one $F$ gauge.

Having defined custodial symmetry breaking, we now we distinguish between two varieties of confinement in a gauge Higgs theory. The first is ordinary color or " $\mathrm{C}$ " confinement, meaning that all particles in the asymptotic spectrum are color neutral. This property holds in both the confinement and Higgs regions. The second we call "separation-of-charge" or " $\mathrm{S}_{\mathrm{c}}$ " confinement [8,9], and this is a generalization of the Wilson loop area law criterion to theories with matter in the fundamental representation of the gauge group. Let us consider, in a pure gauge theory, physical states of the form ( $\Psi_{0}$ is the ground state, $\left.R=|\mathbf{x}-\mathbf{y}|\right)$

$$
\Psi_{V}(R)=\bar{q}^{a}(\mathbf{x}) V^{a b}(\mathbf{x}, \mathbf{y} ; U) q^{b}(\mathbf{y}) \Psi_{0},
$$

where $\bar{q}, q$ are static quark/antiquark operators transforming in the fundamental representation, the $V$ operator transforms like a Wilson line running between sites $\mathbf{x}, \mathbf{y}$, and we normalize

$$
\left\langle\Psi_{0}\left|\operatorname{Tr}\left[V^{\dagger}(\mathbf{x}, \mathbf{y} ; U) V(\mathbf{x}, \mathbf{y} ; U)\right]\right| \Psi_{0}\right\rangle=N
$$

where (in an $\mathrm{SU}(\mathrm{N})$ gauge theory) $N$ is the number of colors. The energy expectation value of this state, above the vacuum energy, is approximated by the lattice logarithmic time derivative

$$
E_{V}(R)=-\log \left[\frac{1}{N}\left\langle\operatorname{Tr}\left[U_{0}^{\dagger}(\mathbf{x}, t) V(\mathbf{x}, \mathbf{y}, t ; U) U_{0}(\mathbf{y}, t) V^{\dagger}(\mathbf{x}, \mathbf{y}, t+1, U)\right]\right\rangle\right] .
$$

The minimum possible energy $E_{\min }(R)$ is the static quark potential, as determined from the behavior of large Wilson loops. Since, in a pure gauge theory, $E_{\min }(R) \sim \sigma R$ at large $R$, and $E_{V}(R)$ is bounded from below by $E_{\min }(R)$, it follows that

$$
\lim _{R \rightarrow \infty} E_{V}(R)=\infty \text { for all } V(\mathbf{x}, \mathbf{y} ; U) \text { operators . }
$$

This is "separation of charge" confinement in a pure gauge theory. It is a stronger condition than C confinement.

Our proposal is that (18) is also the definition of $\mathrm{S}_{\mathrm{c}}$ confinement in gauge Higgs theories, and other gauge + matter theories. Here it is essential that the operator $V(\mathbf{x}, \mathbf{y} ; U)$ depends only on the spacelike link variables, and not on the matter fields, for otherwise one could have, e.g.

$$
V^{a b}(\mathbf{x}, \mathbf{y} ; \phi)=\phi^{a}(\mathbf{x}) \phi^{\dagger b}(\mathbf{y})
$$

which, when inserted in (15), creates two color singlet quark-scalar systems, localized at points $\mathbf{x}, \mathbf{y}$, with a negligible $R$-dependent interaction energy.

It turns out that spontaneous breaking of custodial symmetry, $\langle\Phi\rangle>0$, implies C confinement, while a phase of unbroken custodial symmetry may be either $S_{c}$ confining or massless. To justify 
this statement we consider quantization in the physical $F$ gauges, where field operators $q, \bar{q}, \phi$, operating on the vacuum, create physical states. We begin from the fact that although $F$-gauges remove all local symmetry, they leave some global subgroup of the gauge symmetry unfixed. This global symmetry includes at a minimum, in an $\mathrm{SU}(\mathrm{N})$ gauge Higgs theory, the center subgroup

$$
\phi(\mathbf{x}, t) \rightarrow z(t) \phi(\mathbf{x}, t) \quad, \quad U_{0}(\mathbf{x}, t) \rightarrow z(t) U_{0}(\mathbf{x}, t) z^{\dagger}(t+1), z(t) \in Z_{N} .
$$

which can be spontaneously broken on a time slice. Now unbroken custodial symmetry implies in particular that for an operator $Q(\phi, U)$ which depends only on $\phi$ and the spacelike links $U$ on a time slice,

$$
\left\langle\Psi_{0}|Q(z \phi, U)| \Psi_{0}\right\rangle=\left\langle\Psi_{0}|Q(\phi, U)| \Psi_{0}\right\rangle, z(t) \in Z_{N} .
$$

But if this is true for the center subgroup of custodial symmetry, it is also true for the global remnant gauge symmetry (20), because the action of these transformations on the Higgs field and spacelike links on a time slice is identical. Likewise, broken custodial symmetry implies broken remnant gauge invariance, and this is true in all $F$-gauges.

A physical state is charged if it transforms covariantly under the remnant gauge symmetry. In the spin glass phase of an $\mathrm{SU}(\mathrm{N})$ gauge theory, custodial symmetry is broken, remnant gauge symmetry is broken, and the vacuum state is not a state of definite (zero) charge. This means that the field operators $q, \bar{q}, \phi$, which transform covariantly under gauge transformations, do not create a state of definite charge when acting on the vacuum. This is particularly clear in the broken phase in an $F$ gauge in which $\langle\phi\rangle_{F} \neq 0$. We introduce states

$$
\begin{aligned}
\left|\operatorname{charged}_{\mathbf{x y}}\right\rangle & =\bar{q}^{a}(\mathbf{x}) V^{a b}(\mathbf{x}, \mathbf{y} ; U) q^{b}(\mathbf{y})\left|\Psi_{0}\right\rangle \\
\left.\mid \text { neutral }_{\mathbf{x y}}\right\rangle & =\left(\bar{q}^{a}(\mathbf{x}) \phi^{a}(\mathbf{x})\right)\left(\phi^{\dagger a}(\mathbf{y}) q^{a}(\mathbf{y})\right)\left|\Psi_{0}\right\rangle,
\end{aligned}
$$

and consider $V=V_{F}$ operators, where

$$
V_{F}(\mathbf{x}, \mathbf{y}, t ; U)=g_{F}^{\dagger}(\mathbf{x}, t ; U) g_{F}(\mathbf{y}, t ; U),
$$

and where $g_{F}(\mathbf{x} ; U)$ is the gauge transformation the $F$ gauge. We imagine taking $\mathbf{y} \rightarrow \infty$, leaving either an isolated quark, or a color singlet quark-scalar state, at site $\mathbf{x}$. In the spin glass phase, however, these states have a non-zero overlap, even in the $\mathbf{y} \rightarrow \infty$ limit. Evaluating the overlap in an $F$ gauge where $V=\mathbb{1}$,

$$
\begin{aligned}
\left.\lim _{|\mathbf{x}-\mathbf{y}| \rightarrow \infty}\langle\text { neutral }| \text { charged }\right\rangle & \propto \lim _{|\mathbf{x}-\mathbf{y}| \rightarrow \infty}\left\langle\phi^{\dagger a}(\mathbf{x}) \phi^{a}(\mathbf{y})\right\rangle_{F}=\left\langle\phi^{\dagger a}\right\rangle_{F}\left\langle\phi^{a}\right\rangle_{F} \\
& >0 .
\end{aligned}
$$

This non-zero overlap shows that the "charged" state containing an isolated quark at point $\mathbf{x}$ is not really charged; it has a finite overlap with states created by color singlet operators acting on the vacuum at point $\mathbf{x}$. In the broken phase one cannot distinguish charged from neutral states by their differing transformation properties under the remnant symmetry. It can also be shown that $E_{V_{F}}(R)$ is finite at $R \rightarrow \infty$. So in the spin glass phase there always exist $F$-gauges in which the field operators create finite energy states, and $\langle\phi\rangle_{F}$ is non-zero. The broken phase is therefore not $\mathrm{S}_{\mathrm{c}}$ confining, and it does not contain isolated charged particles in the spectrum that can be distinguished by their gauge transformation properties from color singlet particles. This $\mathrm{C}$ confinement. 
However, within the broken phase there are also $F$-gauges in which $\langle\phi\rangle_{F}=0$. It of course makes no sense to say that the theory is $\mathrm{C}$ confining in one gauge, but not in another. The resolution to this conundrum must be that a state such as $\Psi=g_{F}(\mathbf{x} ; U) \phi(\mathbf{x}) \Psi_{0}$ is orthogonal to the vacuum not because the remnant global gauge symmetry is unbroken, but rather because the $g_{F}(\mathbf{x} ; U) \phi(\mathbf{x})$ operator creates a state of infinite energy compared to the vacuum energy. This is easy to verify in axial gauge, where one can prove that $\langle\phi\rangle_{F}=0$ and $\lim _{R \rightarrow \infty} E_{V_{F}}(R)=\infty$ everywhere in the phase diagram. The underlying reason is that a quark operator in this gauge creates a line of electric flux stretching to infinity, and the energy of of such a line is infinite anywhere in the phase diagram.

In the unbroken phase the vacuum is invariant under the remnant $Z_{N}$ gauge symmetry, and there exists a charged sector, created e.g. by the isolated $q, \bar{q}, \phi$ operators in any $F$-gauge, orthogonal to all neutral states. In fact one can prove that in this phase

$$
\left.\lim _{|\mathbf{x}-\mathbf{y}| \rightarrow \infty}\langle\text { neutral }| \text { charged }\right\rangle=0,
$$

for any choice of $V$ operator in (22). If all charged states are infinite energy, then the symmetric phase is an $\mathrm{S}_{\mathrm{c}}$ confinement phase. If instead there exist finite energy charged states, orthogonal to all neutral states, then charged particles will exist in the spectrum, which implies that the system is neither in a $\mathrm{C}$ confinement nor an $\mathrm{S}_{\mathrm{c}}$ confinement phase; the remaining possibility is a massless phase. Such phases exist in the abelian Higgs model in $3+1$ dimensions [10], and lattice SU(2) gauge Higgs theory in 4+1 dimensions [11].

The conclusion is that the spin glass phase is a phase of broken custodial symmetry, broken global $Z_{N}$ gauge symmetry, and $\mathrm{C}$ confinement. This is the Higgs phase. The phase of unbroken custodial symmetry may be either $S_{c}$ confining or massless, depending on the coupling and spacetime dimension. In the absence of a massless phase, the transition from the unbroken phase to the spin glass phase coincides with the transition from $\mathrm{S}_{\mathrm{c}}$ confinement to ordinary color confinement.

\section{References}

[1] K. Osterwalder and E. Seiler, Annals Phys. 110, 440 (1978).

[2] E. H. Fradkin and S. H. Shenker, Phys.Rev. D19, 3682 (1979).

[3] J. Frohlich, G. Morchio, and F. Strocchi, Nucl. Phys. B190, 553 (1981).

[4] G. 't Hooft, NATO Sci. Ser. B 59, 117 (1980).

[5] W. Caudy and J. Greensite, Phys.Rev. D78, 025018 (2008), arXiv:0712.0999.

[6] J. Greensite and K. Matsuyama, (2020), arXiv:2001.03068.

[7] S. F. Edwards and P. W. Anderson, Journal of Physics F: Metal Physics 5, 965 (1975).

[8] J. Greensite and K. Matsuyama, Phys. Rev. D96, 094510 (2017), arXiv:1708.08979.

[9] J. Greensite and K. Matsuyama, Phys. Rev. D98, 074504 (2018), arXiv:1805.00985.

[10] J. Ranft, J. Kripfganz, and G. Ranft, Phys. Rev. D28, 360 (1983).

[11] B. B. Beard et al., Nucl. Phys. Proc. Suppl. 63, 775 (1998), arXiv:hep-lat/9709120. 